\title{
A Study on Marginal Continuty of Shoulder and Chamfer Finish Line of Metal Crown
}

\author{
Dr. Mahenaz Munira1, Dr. Faruk Nahar², Dr. Nasima Khatun³, \\ Dr. Mahamuda Khatun ${ }^{4}$, Dr. Alia Sultana ${ }^{5}$
}

Received: 01 October $2016 \quad$ Accepted: 12 November 2016

\section{Abstract:}

Background: Marginal continuty is very important for the longevity of restoration. The junction between the cemented restoration and the tooth is always a potential site for recurrent caries, gingivitis and periodontitis and ultimately failure of crown as well as teeth. Hypothesis: Marginal continuity for shoulder finish line for anterior teeth and chamfer for posterior teeth are equally effective. Objectives: To evaluate the marginal continuity of metal crown. Methods: Thirty extracted human teeth were prepared with shoulder margin (group-A) and another thirty teeth were prepared with chamfer (group-B) as methodically. Metal crown was fabricated by lost wax technique of casting procedure. All of the crowns (both group- $A$ and group-B) were cemented by glass inomer luting cement. Each crown was sectioned in faciolingual direction at the midpoint of each surface by slow speed isomet saw. The sectioned crowns were examined under sreriomicroscope for measurement of marginal continuty. Data were collected on the basis of marginal continuty of each crown was compared. Collected data were processed and analyzed using SPSS (Statistical Package for Social Service) version 12. Results: The frequency distribution of marginal continuity were statistically non significant between two groups. Conclusion: All metal crowns had greater continued margin than discontinued margin.

\section{Introduction:}

The portion of tooth which is covered by enamel is known as crown. There are three types of crown: anatomic crown, clinical crown, artificial crown. Portion of natural tooth that extends from its cement-enamel junction to the occlusal surface or incisal edge is called anatomic crown. The portion of natural tooth that extends from the bottom of the sulcus to the occlusal surface or incisal edge is called clinical crown. The cement-enamel junction of teeth is called margin. Marginal accuracy is considered a crucial factor in the success and longevity of a cast restoration. Ideally, cemented cast restoration margin should precisely continuously meet the finish line of the prepared teeth with non-detectable junctions. Practically clinical perfection is difficult to achieve and difficult to verify ${ }^{1}$. A cast crown is considered satisfactory when is presents suitable anatomical form, correct polishing and adequate marginal adaption ${ }^{2}$. Successes depend on the marginal adaption of the casting, the surface of the margins and the luting cement used. The accuracy of the casting such as continued margin will be determined by the preciseness of the previous construction procedure such as the impression, the construction of the gypsum die, the waxing technique, the investment technique and the casting procedure ${ }^{3}$.

The objective of luting cement is to fix the casting to the preparation and to seal the gap between the crown and preparation. One of the greatest shortcomings of dental luting cements is high solubility rate and their permeability. The excellence of the finish line depends on the accurate finish of the preparation and accurate casting procedure ${ }^{3}$. The presently used preparation margins are shoulder, chamfer, shoulder bevel, knife edge, slope shoulder etc. The requirements of preparation are minimum width of $0.5 \mathrm{~mm}$ for prepared margins, bevel angulations between 30 degrees and 45 degrees for optimum margin closure, smooth surface on

1. BDS, MS, Assistant Professor, Shaheed Suhrawardy Medical College Hospital, Dhaka.

2. BDS, MS, Assistant Professor, Shaeed Suhrawardy Medical College, Dhaka.

3. BDS, MS, Assistant Professor, Sir Salimullah Medical College, Dhaka.

4. BDS, Lecturer, Shaheed Suhrawardy Medical College, Dhaka.

5. BDS, DDS, MDS, Chairman, BSMMU.

Correspondence : Dr. Mahenaz Munira, email: munira78@gmail.com, cell: 01715388055 
prepared margins, preparation without overextension or unsupported enamel and easy to identification in the impression or on the die.

Smooth prepared margins can be identified easily on the die. Rough prepared margins may cause incomplete fit of crown, increasing the chance of cement solubility. After crown cementation, the cavosurface angle of the margin may not be covered by cement. The margin must be as smooth as possible to reduce accumulation, vitro studies can help to estimate the marginal adaption of different type of margin ${ }^{1}$.

Marginal fit as well as marginal continuity is very important because the junction between the cemented restoration and the tooth is always a potential site for recurrent caries due to the dissolution of luting agent and the inherent roughness. If the adaption of restoration of teeth is more accurate, lesser the chance of recurrent caries or periodontal disease, ultimately the longevity of the restoration is increased $^{5}$. Special measurements of these gaps can be compared to the theoretical zero. Minimal dimension for the gaps were not determined clinically; consequently there are no existing standard for measuring these gaps ${ }^{5}$. The study evaluate the marginal fit of shoulder finish line for anterior teeth and chamfer for posterior teeth. In this study human teeth were selected because human teeth are usually restored by easily available metal alloy or composite resin. The properties of most artificial substitutes are not often as those of human teeth ${ }^{4}$. Nickel-chromium-Beryllium (NiCrBe) alloy was used because of its biocompatibility, high mechanical strength, less clinical inconvenience, satisfactory resistance to corrosion.

\section{Materials \& Methods}

This observational in vitro study was carried out in the Department of Prosthodontics, Faculty of Dentistry, Bangabandhu Sheikh Mujib Medical University (BSMMU), Dhaka; Department of Geology, Dhaka University, Dhaka and Material Science Division, Atomic Energy Centre, Dhaka. Freshly extracted human anterior and posterior teeth were divided into 2 groups each containing 30 teeth. In Group A: This group consisted of maxillary anterior teeth with shoulder margin. In Group B: This group consisted of maxillary posterior teeth with chamfer margin. Well formed healthy and freshly extracted human maxillary teeth are selected for the study.

Research instruments and materials:

Sterio Microscospe, Slow speed isomet saw,
Alginate, Dental stone, Glass-Inomer cement, $\mathrm{NiCrBe}$ Alloy.

\section{Clinical Procedure:}

The teeth selected were immersed in $1 \%$ sodium hypochlorite solution in a screw capped glass vial for two days to remove any organic debris from the root surface as well as for preservation. All teeth were carefully cleaned to eliminate tartar, calculus, stain and remained tissue with the help of ultrasound scaler. Then the specimens were again immersed in $1 \%$ sodium hypochlorite for 24 hours. After 24 hours, the cleaned teeth were stored in normal saline prior to tooth preparation for the study. Tooth preparation was carried out methodically. Then all teeth were immersed in normal saline.

\section{Tooth Preparation:}

The reduction of teeth was done by following biological \& mechanical consideration. Enamel was reduced from all surfaces of teeth for metal crown.

Facial reduction: Depth orientation grooves were placed on the labial and incisal surface with the flat end tapered diamond. The grooves were $1.2 \mathrm{~mm}$ deep on the labial surface and $2 \mathrm{~mm}$ deep in the incisal edge. The labial grooves were cut with the diamond held parallel to the gingival one third of the labial surface. A second set of two grooves was made parallel to the incisal two third of the uncut labial surface. The labial surface of all metal preparation is done in two planes to achieve adequate clearances for good esthetics without encroachment of the pulp. The gingival portion of labial surface is reduced to 1.2 $\mathrm{mm}$ depth by a flat end tapered diamond bur. This reduction extends around the labioproximal line angles and faded out on the lingual aspect of the proximal surface. The end of the flat end tapered diamond bur will form the shoulder finish line while the axial reduction is done with the side of the diamond. The shoulder margins were minimized to $1.0 \mathrm{~mm}$ wide.

Incisal reduction: The reduction of incisal edge /occlusal surface was done about $2 \mathrm{~mm}$ for adequate metal thickness.

Lingual reduction: Lingual reduction was done carefully with the small wheel diamond. Because over reduction of the junction between the cingulam and the lingual wall was necessary for the retention of the crown.

Proximal Reduction: Using the round end diamond, proximal reduction was done.

Finish line preparation: For anterior teeth, $1 \mathrm{~mm}$ 
shoulder margin was done by flat end tapered diamond and for posterior teeth, chamfer margin was done by torpedo diamond.

Finishing: All axial walls were smoothened and all sharp line angles were rounded. Care was taken not to create undercut in the axial walls where they join the finish line.

\section{Laboratory Procedure:}

Impression of the prepared teeth were taken by irreversible hydrocolloid impression material alginate (lygin, dentamerica, USA) using stock tray. Working cast and dies were fabricated with the stone. Preparation of dies \& metal crowns were made by lost wax technique. Then all metal crowns were cemented onto their respected teeth by Glass-lonomer luting cement. Then each crown was sectioned with a diamond wafering saw in facio-lingual direction at the midpoint of each tooth. Every specimen was examined under a scanning Electron microscope for measurement of marginal gap. Measurement of marginal gap was determined at the facial/buccal and palatal midpoint of each crown. Available data were analyzed statistically. Chi square test and student's t test were performed to detect statistical significance of the study. $P$ value $<0.05$ was accepted as significant.

\section{Results}

Frequency distribution of marginal continuity in both groups $(\mathrm{n}=60)$

In group A , 24(80\%) sample had continued margin and $6(20 \%)$ sample had discontinued margin .In group B , 18(60\%) had continued margin and 12 $(40 \%)$ sample had discontinued margin .In both subdivision of marginal continuity, the difference in frequency (\%) between two groups were statistically non significant.

\begin{tabular}{|c|c|c|c|c|c|}
\hline $\begin{array}{l}\text { Marginal } \\
\text { continuity }\end{array}$ & $\begin{array}{c}\text { Group } \\
\text { A } \\
n=30\end{array}$ & & $\begin{array}{c}\text { Group } \\
\text { B } \\
n=30\end{array}$ & & P value \\
\hline & $\mathrm{N}$ & $\%$ & $n$ & $\%$ & \\
\hline $\begin{array}{l}\text { Continued } \\
\text { margin }\end{array}$ & 24 & 80.0 & 18 & 60.0 & $0.090^{* *}$ \\
\hline $\begin{array}{l}\text { discontinu } \\
\text { ed margin }\end{array}$ & 6 & 20.0 & 12 & 40.0 & $0.090^{\text {ns }}$ \\
\hline
\end{tabular}

ns $=$ non significant, $\mathrm{n}=$ Total number of subjects

Data were analyzed using chi square test. Group $A=$ shoulder margin, Group B = chamfer margin.

\section{Discussion}

This In vitro study was conducted to evaluate the marginal continuity of shoulder margin and chamfer margin for metal crown. Marginal continuity was observed among all the crowns of this study. For the evaluation, marginal continuity was categorized into continued margin and discontinued margin. On the basis of frequency of marginal continuity of crown, shoulder finish line of anterior teeth had higher frequency than that of chamfer finish line of posterior teeth for continued margin. Shoulder finish line of anterior teeth had lower frequency than that of chamfer finish line of posterior teeth for discontinued margin. Blackman stated in his study, marginal fit of metal crown which is made of pure titanium. The present study made similar observation. Petteno stated that marginal fit of crown by using three different metal ceramic systems that were composite alloy, electroforming and high noble alloy. The present study made dissimilar observation because the material used in this study was different. Rosentrit8 stated that marginal adaptation of crowns which was made by ceramic and fixed on natural and artificial teeth. The present study made similar observation because natural teeth Wazzan ${ }^{1}$ states that the marginal and internal fit of crown which was made by commercially pure titanium (CPTi) and titanium-aluminium-vanadium (Ti-6Al-4V) alloy cast restorations ${ }^{1}$. The present study made similar observation through the material used in this study which was nickel chromium beryllium (Ni-Cr-Be) alloy ${ }^{1}$. Petro ${ }^{2}$ stated that marginal and internal discrepancy of complete cast crown by using different casting method ${ }^{2}$. In various studies marginal continuity measured and reported in varieties of way. Different margin, impression materials, dental stone, investment materials, casting procedure, luting agent, alloy were chosen. In this study alginate, type IV dental stone, conventional casting technique and $\mathrm{NiCrBe}$ alloy was selected because these were randomly used in Bangladesh. Every step should be done with great care and perfectly to avoid discontinuity as well as misfit.

\section{Conclusion}

After completion of the study, shoulder finish line of anterior teeth and chamfer finish line of posterior teeth of metal crown were successfully adapted. All metal crowns had greater continued margin than 
discontinued margin.
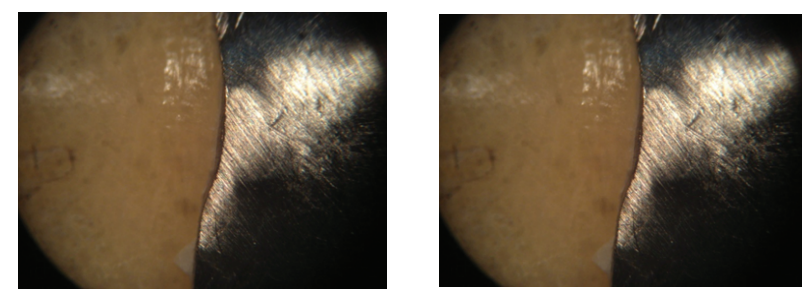

Fig :Continued margin of metal crown.
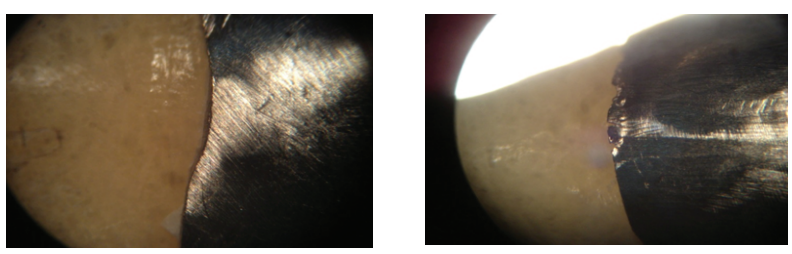

Fig : Discontinued margin of metal crown.

\section{Bibliography}

1. Wazzan KAA, Nazzawi AAA. Marginal and internal adaptation of commercially pure titanium and titanium-aluminium- vanadium alloy cast restorations. The Journal of contemporary Dental Practice .2007; 8(1): 19-23.

2. Preto $R$.Influence of casting methods on marginal and internal discrepancies of complete cast crowns. Brazilian Dental Journal.2004;15.

3. Theuniers G, Clercq MD.Finishingprocedure for the preparation of crown margins.Journal of Prosthetic Dentistry. $1987 ; 58(5): 545-51$.

4. Rosentritt $M$ et al .In vitro fracture force and marginal adaptation of ceramic crowns fixed on natural and artificial teeth. International Journal of Prosthodontics.2000;13(5) : 387-391.

5. Holmes JR, Sulik WD, Holland GA, Bayne SC. Marginal fit of castable ceramic crowns.Journal of Prosthetic Dentistry.1992;67 (5): 594-595.

6. Blackman R, Baez R, Barghi N. Marginal accuracy and geometry of cast titanium copings. Journal of Prosthetic Dentistry. 1992;67 (4) :435-440.

7. Petteno D .Comparison of marginal fit of three Different Metal -ceramic Systems : An In Vitro Study. International Journal of Prosthodontics. 2000 ;13(5):405-408. 